\title{
LA PLANIFICACIÓN Y GESTIÓN TERRITORIAL INTEGRAL EN ISLAS PEQUEÑAS COMO HERRAMIENTA DE REDUCCIÓN DE RIESGOS NATURALES
}

\section{Comprehensive Territorial Management And Planning In Small Islands As A Tool For Natural Hazard Reduction}

\author{
Mario J. Gallego Cosme \\ Universidad Nacional de Educación a Distancia, España. \\ mgallego136@alumno.uned.es
}

\begin{abstract}
Resumen: La vulnerabilidad de los espacios insulares de tamaño pequeño deviene de su falta de recursos de todo tipo, incluidos los humanos, lo que en ocasiones convierte a sus habitantes en sujetos pasivos de los riesgos naturales y otras contingencias negativas, sobre todo de tipo económico y social. En este sentido, la escasez de espacio incide directamente en el progreso y bienestar de estos territorios, que deben abordar la ardua tarea de encontrar un modelo de desarrollo sostenible que garantice su crecimiento económico de manera autónoma a largo plazo sin comprometer el medio ambiente. Este artículo analizará la necesidad de lograr una gestión y planificación integral del territorio insular como medio para lograr estos fines y, sobre todo, como principal herramienta para la reducción de la vulnerabilidad de estos espacios pequeños en materia de riesgos naturales.
\end{abstract}

Palabras clave: Calentamiento global, islas pequeñas, ordenación del territorio, vulnerabilidad. 


\begin{abstract}
Vulnerability of small island spaces comes from their lack of resources of every kind, including human ones, which sometimes turns them into passive subjects of natural hazards and other negative contingencies, particularly those of economic and social nature. In this regard, the shortage of space directly affects the progress and welfare of these territories, which must address the difficult task of finding a model of sustainable development that ensures an autonomous economic growth over the long term without compromising the environment. This paper will discuss the need for a comprehensive planning and management of the island territories as a means to achieve these ends and, above all, as the main tool for reducing the vulnerability of these small spaces of natural hazards.
\end{abstract}

Keywords: Global warming; small islands; territorial planning; vulnerability.

Fecha de recepción: 05-12-12. Fecha de reenvios 23-09-13, 30-12-13, 27-03-14 Fecha de aceptado: 01-04-14. Fecha de publicación: 22-04-14.

http://dx.doi.org/10.15359/abra.34-48.1

\title{
La vulnerabilidad de las islas pequeñas
}

Que las islas pequeñas son espacios especialmente vulnerables es un hecho incontestable, pues la escasez de superficie constituye un limitante de primer orden que influye decisivamente en aspectos de gran importancia como el desarrollo económico o una gestión pública efectiva por parte de los gobiernos. Esta vulnerabilidad, entendida como el grado al cual un sistema es sensible e incapaz de hacer frente a las adversidades (Nurse y Sem, 2001, p. 865), está fundamentada en dos aspectos inherentes a estos espacios: su condición de territorio de escaso tamaño y el factor insular que los caracteriza. 
Tabla 1

Estados insulares pequeños y en desarrollo según las Naciones Unidas.

\begin{tabular}{|c|c|c|c|c|c|}
\hline & $\begin{array}{l}\text { Área } \\
\left(\mathrm{Km}^{2}\right)\end{array}$ & Poblacion & & $\begin{array}{l}\text { Área } \\
\left(\mathrm{Km}^{2}\right)\end{array}$ & Población \\
\hline Antigua y Barbuda & 442 & 68.320 & Maldivas & 298 & 339330 \\
\hline Bahamas & 13.940 & 347.200 & Mauricio & 2.040 & 1.306 .600 \\
\hline Bahrein & 665 & 1.248 .348 & Nauru & 21 & 10.300 \\
\hline Barbados & 431 & 273.900 & Palaos & 459 & 20.600 \\
\hline Belice* $^{*}$ & 22.696 & 327.719 & $\begin{array}{l}\text { Papua- Nueva } \\
\text { G. }\end{array}$ & 462.840 & 7.013 .800 \\
\hline Cabo Verde & 4.033 & 500.600 & Samoa & 2.831 & 183.900 \\
\hline Comores & 2.170 & 753.900 & $\begin{array}{l}\text { San Cristóbal } \\
\text { y N. }\end{array}$ & 261 & 53.100 \\
\hline Cuba & 110.861 & 11.253 .700 & $\begin{array}{l}\text { San Vicente } \\
\text { y G. }\end{array}$ & 388 & 109.400 \\
\hline Dominica & 754 & 67.700 & Santa Lucía & 622 & 176.000 \\
\hline E. F. Micronesia & 702 & 111.500 & $\begin{array}{l}\text { Santo Tomé } \\
\text { y P. }\end{array}$ & 964 & 190.428 \\
\hline Fiyi & 18.270 & 868.400 & Seychelles & 455 & 86.900 \\
\hline Granada & 344 & 104.900 & Singapur & 618 & 5.187 .900 \\
\hline Guinea-Bissau* & 36.125 & 1.547 .100 & Surinam & 163.265 & 529.400 \\
\hline Guyana* & 214.696 & 756.000 & $\begin{array}{l}\text { Rep. } \\
\text { Dominicana }\end{array}$ & 48.511 & 10.056 .200 \\
\hline Haití & 27.750 & 10.123 .800 & Timor Oriental & 15.007 & 1.153 .800 \\
\hline Is. Marshall & 181 & 68.480 & Tonga & 650 & 104.500 \\
\hline Is. Salomón & 28.896 & 552.300 & Trinidad y $\mathrm{T}$. & 5.130 & 1.346 .400 \\
\hline Jamaica & 10.990 & 2.751 .300 & Tuvalu & 26 & 9.800 \\
\hline Kiribati & 726 & 101.100 & Vanuatu & 12.189 & 245.600 \\
\hline
\end{tabular}

Nota: Fuente: elaboración propia con datos de la página web de UN-OHRLLS ${ }^{1}$ (2013). Los tres países señalados con asterisco $\left(^{*}\right)$ no son islas.

Aunque no existe acuerdo sobre qué es una isla pequeña, pues resulta complicado categorizar el tamaño de un territorio con un criterio ampliamente aceptado, podría establecerse que entrarían en esta categoría muchos de los 38 Estados insulares y 19 territorios

1 Oficina del Alto Representante para los Países Menos Adelantados, los Países en Desarrollo sin Litoral y los Pequeños Estados Insulares en Desarrollo. 
dependientes listados por la Organización de las Naciones Unidas como "pequeños Estados insulares en desarrollo" (SIDS, por sus siglas en inglés), además de cuantas islas compartan atributos similares de población o tamaño con este grupo, aunque formen parte de la estructura fundamental de otros Estados.

En realidad, la disparidad de territorios mencionados pone de manifiesto que se trata de un listado abierto y que mayormente permite dar idea del conjunto de islas que quedan clasificadas como pequeñas, pues si atendemos a la tabla 1, que muestra los Estados que la ONU considera como "pequeños y en desarrollo", se pueden apreciar algunas ausencias (Malta o Chipre serían claros ejemplos) o incluso la presencia de países no insulares. En cualquier caso, es posible establecer una serie de características comunes a, prácticamente, todas las islas de tamaño pequeño. Por consiguiente, se trata de espacios insulares pequeños, escasos en población y recursos naturales; con economías poco diversificadas y tendencia a una alta dependencia externa, inclusive en materia alimentaria; además, en ocasiones se trata de islas asoladas o mal comunicadas y susceptibles a las amenazas ante riesgos naturales; y con malas infraestructuras y cierta falta de recursos humanos capacitados. Estas condiciones también las cumplen la mayoría de los espacios insulares no autónomos del mundo (pues casi todos son más pequeños que la mayoría de los Estados insulares independientes de la lista) y muchas islas vulnerables que forman parte de la estructura fundamental de otros países.

La diferenciación en cuanto al grado de soberanía y autonomía de los territorios es clave, pues de este factor dependerá qué administración se deberá hacer cargo de la planificación y gestión de estos espacios. En el caso de los Estados, ellos mismos son los máximos responsables de su gobierno. Los territorios dependientes (tabla 2), como norma general, reciben asistencia de los Estados de los que están tutelados, aunque la fórmula del autogobierno (financiado o no) en estos asuntos es muy común. Por el contrario, las islas integradas en cualquier país, normalmente estarán subordinadas a otras entidades administrativas, aunque la casuística es muy variada. En el presente artículo la atención se centrará sobre todo en las islas menos pobladas, más pequeñas, o aquellas en las que la configuración física o geográfica incide en su fragilidad (islas alargadas, demasiado montañosas, con poca altitud 
respecto al nivel del mar, atolones, etcétera), sea cual sea la principal administración responsable de su gestión.

Tabla 2

Territorios dependientes insulares pequeños y en desarrollo según las Naciones Unidas.

\begin{tabular}{|c|c|c|c|c|c|}
\hline & Área (Km2) & Población & & Área (Km2) & Población \\
\hline Anguila & 102 & 15.423 & $\begin{array}{l}\text { Is. Vírgenes } \\
\text { (RU) }\end{array}$ & 153 & 31.148 \\
\hline Aruba & 193 & 107.635 & Martinica & 1.128 & 401.000 \\
\hline Bermuda & 54 & 69.467 & Montserrat & 102 & 5.164 \\
\hline Curaçao & 444 & 152.760 & Niue & 260 & 1.269 \\
\hline Guam & 549 & 159.358 & $\begin{array}{l}\text { Nueva } \\
\text { Caledonia }\end{array}$ & 19.060 & 260.166 \\
\hline Guadalupe & 1.705 & 463.000 & $\begin{array}{l}\text { Polinesia } \\
\text { Francesa }\end{array}$ & 4.167 & 274.512 \\
\hline Is. Cayman & 264 & 55.036 & Puerto Rico & 9.104 & 3.897 .960 \\
\hline Is. Cook & 236 & 10.777 & $\begin{array}{l}\text { Turcas y } \\
\text { Caicos }\end{array}$ & 616 & 39.000 \\
\hline $\begin{array}{l}\text { Is. Marianas } \\
\text { del N. }\end{array}$ & 477 & 44.582 & $\begin{array}{l}\text { Samoa } \\
\text { Americana }\end{array}$ & 199 & 68.061 \\
\hline $\begin{array}{l}\text { Is. Vírgenes } \\
\text { (EEUU) }\end{array}$ & 352 & 109.174 & & & \\
\hline
\end{tabular}

Nota: Fuente: elaboración propia con datos de la página web de UN-OHRLLS (2013).

La mayor parte de la población del mundo está concentrada en zonas costeras que representan un $10 \%$ de la superficie total del planeta (Hinrichsen, 1998). En las islas de tamaño pequeño la proporción de habitantes que vive en las cercanías de un litoral marítimo es muy alta, y de hecho, se estima que "los mayores asentamientos de las islas pequeñas están localizados no más alejados de uno o dos kilómetros de la costa" (Nurse, McLean \& Suarez, 1997, sección 9.3.7.1). Evidentemente, los efectos de esta situación son de enorme importancia por diversos motivos. El atractivo que históricamente han tenido las áreas litorales en nuestro planeta presenta bastantes ventajas para la administración territorial, derivadas de la concentración poblacional, y que son igualmente muy positivas para cualquier isla de tamaño pequeño, como son la 
maximización en los servicios y mejoras en los transportes o la creación de economías de escala.

Buena parte de los recursos naturales y culturales más valiosos de la población, para la utilización y las actividades económicas se encuentran en el ámbito litoral, aunque por este motivo, también en este espacio ocurren la mayoría de los problemas y los conflictos. Por lo tanto, la presión en los litorales conlleva igualmente importantes consecuencias negativas que amenazan los mencionados beneficios (Creel, 2003, p.1), y que sin duda pueden tener efectos muy adversos para las islas.

Para las islas el desarrollo autónomo y sostenible es fundamental, sin embargo, encontrar el equilibrio entre el respeto al medio y su explotación en estos espacios reducidos es muy complicado. Lo ideal es la consecución de un sistema de gestión sostenible que debe estar basado en un uso de los recursos de forma que se garantice indefinidamente un aprovechamiento constante de estos: el recurso no se agota, ni se aprovecha en exceso, ni se explota antes de que haya madurado (Fernández, 2011, pp. 24-26). Obviamente, lograr este objetivo es complicado, máxime cuando desde el punto de vista económico, preocupa que la sostenibilidad implique estaticidad en la producción, aunque a gran escala, realmente, y tras un período de tiempo dilatado, el rendimiento sostenible tenderá a producir efectos positivos, más allá de resultados inmovilistas (Gómez, 2002).

En este punto, es necesario destacar que a nivel internacional ha sido en los últimos años cuando las islas de tamaño pequeño han empezado a dotarse de instrumentos que abordan sus preocupaciones en la materia. Aunque en la actualidad existen algunas Organizaciones regionales que, de alguna manera, se ocupan de estos temas (por ejemplo el Pacific Islands Forum Secretariat, la Comisión del Océano Índico o el CARICOM) sus actividades se circunscriben de forma exclusiva a sus ámbitos geográficos, si bien la producción científica de cada una de ellas ha podido ser aplicada en otros casos. La única estructura verdaderamente internacional con vocación global (a la sazón emanada a instancias de la ONU en 2001) es la Oficina del Alto Representante para los Países Menos Adelantados, los Países en Desarrollo sin Litoral y los Pequeños Estados Insulares en Desarrollo, aunque es necesario destacar que su acción se ha concentrado en el acopio de la producción científica, la visibilidad de las problemáticas insulares internacionalmente y las 
recomendaciones consultivas. Dicho de otro modo, no existe ninguna estructura supranacional que se encargue de coordinar e instrumentalizar la planificación territorial de todas las islas de tamaño pequeño del mundo, lo cual resulta lógico si se tiene en cuenta el funcionamiento del sistema internacional en conjunto, ya que este se fundamenta en la voluntariedad de las normas.

\section{Principales amenazas para las islas de tamaño pequeño}

Las amenazas ante riesgos naturales son rasgos que ponen en riesgo el bienestar o la vida humana por las consecuencias catastróficas que pueden llegar a producir. Las más frecuentes son de origen geológico (seísmos, erupciones volcánicas, movimientos de ladera, etc) y climático (inundaciones, granizo, olas de calor, etc.), aunque existen otras de origen antrópico en su mayoría. Los daños que estos fenómenos llegan a producir pueden ser cuantiosos, inclusive en términos no materiales, como en el caso de la pérdida de vidas humanas.

Mediante un análisis realizado para cada territorio, los planes de ordenación territorial deben contemplar la posibilidad de que estas amenazas puedan ocurrir. El objetivo es proteger a la población y aminorar los daños en caso de que estos se produjeran. Las actuaciones más habituales frente a estas amenazas de riesgos naturales son entre otras: la construcción de infraestructuras para mitigar las mismas (drenaje, plantación de árboles, acondicionamiento de cauces), así como la creación de sistemas de previsión y vigilancia (redes de vigilancia sísmica, volcánica e hidrológica). En el caso de las islas, uno de los principales vectores de vulnerabilidad deviene del hecho de la gran densidad poblacional que se aglomera en las cercanías costeras.

No obstante, los efectos del llamado calentamiento global son los que mayor y más grave problema pueden representar para las islas pequeñas a mediano plazo. Las consecuencias más importantes e inmediatas para los pequeños territorios insulares "estarán relacionadas con los cambios en el nivel de los mares, los regímenes de lluvias, los balances de humedad del suelo, los vientos predominantes (velocidad y dirección) [...] variaciones regionales en los patrones de oleaje" (Hay, 2000, p. 285) y fenómenos derivados de los anteriores tan relevantes como la intrusión 
salina en acuíferos. Muchas de estas consecuencias, obviamente, serían devastadoras, pues la mayoría de estas tienen relación con el agua, que es un recurso igualmente escaso en muchas islas pequeñas y que afecta directamente a la vida de las personas, a la industria y a la agricultura. El hecho es que muchos de estos territorios "únicamente tienen una fuente de abastecimiento de agua, como las reservas subterráneas (Barbados, Antigua, Bahamas o Kiribati), el agua de lluvia (Tuvalu, Maldivas,etc.), depósitos en superficie e importaciones (Singapur), o ríos y otros cauces fluviales (Seychelles y Dominica)" (Nurse y Sem, 2001, p., 860).

Sin embargo, indudablemente, el efecto más pernicioso del cambio climático para las islas es el aumento del nivel del mar, el cual principalmente está afectando a países con poca altitud como Bahamas, Antigua y Barbuda (ver figura 1), Seychelles, entre otros. Por ejemplo el archipiélago de Maldivas, que literalmente, se encuentra en grave peligro de desaparición bajo las aguas del Índico. Aunque no es cometido de este artículo el análisis pormenorizado del problema del calentamiento global y su influencia en la elevación de las aguas oceánicas, por su relevancia, merece la pena detenerse en las opciones que tienen los territorios implicados en tan inexorable hecho. En el momento en que se llega a cierto punto de "no retorno", las alternativas para los habitantes son básicamente tres: la protección, la acomodación y la retirada. Probablemente, lo ideal sea una combinación de las tres (Nicholls, y Mimura, 1998, p. 14), aunque, por razones evidentes, no siempre esto es posible.

La protección ante la elevación de las aguas puede llevarse a cabo mediante la preservación de barreras naturales (como los bosques litorales, los arrecifes de corales o los manglares) o abordando la construcción de obras de ingeniería que actúen como barrera. Ciertamente, estas opciones podrían utilizarse conjuntamente y nada impide su implantación como medida previsora o mitigadora, sobre todo en el caso de las naturales. Hay que decir que es preciso tener en cuenta que en algunos casos "las obras de ingeniería podrían no llegar a ser la mejor opción, ya que en ocasiones han incidido en el problema que pretendían resolver" (Nurse y Sem, 2001, p. 857), sobre todo como resultado de diseños ineficaces. Un ejemplo de esto, estudiado por Mimura y Nunn (1998, p. 41) en la isla de Fiyi, es el caso de muros verticales fabricados con materiales muy impermeables obtenidos normalmente en las cercanías de las zonas costeras, pero que terminan 
por colapsar debido a la erosión causada por las olas en la base de estas construcciones y que en su caída arrastran parte del terreno que se intentaba preservar de daños.

La acomodación básicamente consiste en elevar el suelo por medio de la transferencia de materiales hacia el lugar que se intenta preservar. Es realmente una medida que tiende a ser más costosa que la anterior, aunque en determinados casos es la única alternativa posible, sobre todo cuando el coste (económico y social) de la retirada no puede afrontarse. Además, como se actúa sobre el territorio que se intenta proteger, debe realizarse escalonadamente o por partes, lo cual ralentiza enormemente el proceso. Sin embargo, al igual que en el caso de las medidas de protección, podría ocurrir que no se lograse el resultado previsto o que simplemente se retrase un tiempo lo inevitable.

La retirada se basa en la existencia de un lugar más propicio y elevado hacia el cual se deberá relocalizar la población en riesgo. Si este lugar existe y se puede asumir lo que implica la mudanza de toda la población afectada a ese lugar, probablemente esta sea la mejor opción, aunque es innegable que son muchas las dificultades que un proyecto de tal magnitud entraña.

De cualquier modo, si se realiza de manera planificada y en fases que permitan un gasto asequible en el tiempo, una buena alternativa en el caso de las islas grandes que no puedan poner en ejecución las otras opciones, es marcharse de forma ordenada (Leatherman, 1997). Probablemente esta sea la alternativa más cara, pero no cabe duda de que es la más sencilla de llevar a cabo cuando no se dispone de la tecnología requerida para la protección o la acomodación y además si se cuenta con un lugar propicio para la relocalización.

Sin embargo, por tamaño, "para islas pequeñas estas medidas no suelen tener una verdadera utilidad práctica" (Nurse y Sem, 2001 p. 856). Asimismo se debe tener en cuenta que, ante el peor escenario posible, la retirada sería la única opción posible si la situación lo requiere, aunque quedaría por resolver la incógnita del lugar de relocalización, sobre todo en el caso de Estados insulares independientes. Cada Administración deberá realizar un cálculo que, incluso por circunstancias emotivas, nunca será fácil, pero se necesita contar con un correcto inventario territorial que permita una toma de decisiones adecuada. De esta manera, 
cada país decidirá en función de las características de su territorio y demás variables sobre la mejor forma de afrontar una contingencia que compromete de forma tan directa su futuro. En el caso de archipiélagos, "podría ser necesario el sacrificio de algunas islas si con ello la nación entera se consigue salvar de la inundación" (Nurse y Sem, 2001, p. 857).

\section{La gestión y planificación integrada de los espacios insulares}

Resulta indispensable resaltar que la urgencia con la que se han venido requiriendo las medidas adaptativas en las islas de tamaño pequeño ha provocado un gran incremento de medidas inconexas y proyectos descoordinados, y no planes estratégicos encaminados al largo plazo y sincrónicos (Mimura y Nurse, 2007, p. 709). Por lo tanto, se hace necesaria una gestión y planificación integrada de los espacios insulares con especial atención al ámbito litoral, en la que se tengan en cuenta diversos puntos de vista como el administrativo, el geográfico, el funcional y el social (Barragán-Muñoz, 2004).

El objetivo básico de esta gestión y planificación integral consiste en resolver aquellas contingencias que amenacen la integridad y permanencia de los recursos al mismo tiempo que se favorece el desarrollo social y económico. En este sentido, esta propuesta de planeamiento y gestión integrada requiere de un enfoque que combine las dos escalas de trabajo requeridas, las cuales son: la isla como conjunto sujeto a una ordenación global (incluyendo las aguas que caigan bajo su soberanía) y el ámbito litoral como clave por ser también este un espacio urbano en la mayoría de los casos.

Por otra parte, sería muy útil que esta gestión y planificación integrada del territorio se lleve a cabo bajo el paradigma del "ciclo de mejora continua" (Doménech y Sanz, 2010, p. 17), la cual radica en el establecimiento de un sistema cíclico de fases que comience por el necesario diagnóstico inicial y termine con una fase de seguimiento y revisión del sistema una vez que se ha llevado a cabo la planificación y la ejecución. 


\section{La isla como conjunto sujeto a una ordenación global}

La consideración de una escala insular global de planeamiento y gestión sería un elemento prácticamente indispensable para la necesaria coordinación entre administraciones, pues facilita que los diferentes planeamientos sectoriales se realicen de una manera armonizada mediante un plan general de actuación de política pública. El fundamento de este enfoque recae en la constatación de que en los espacios insulares

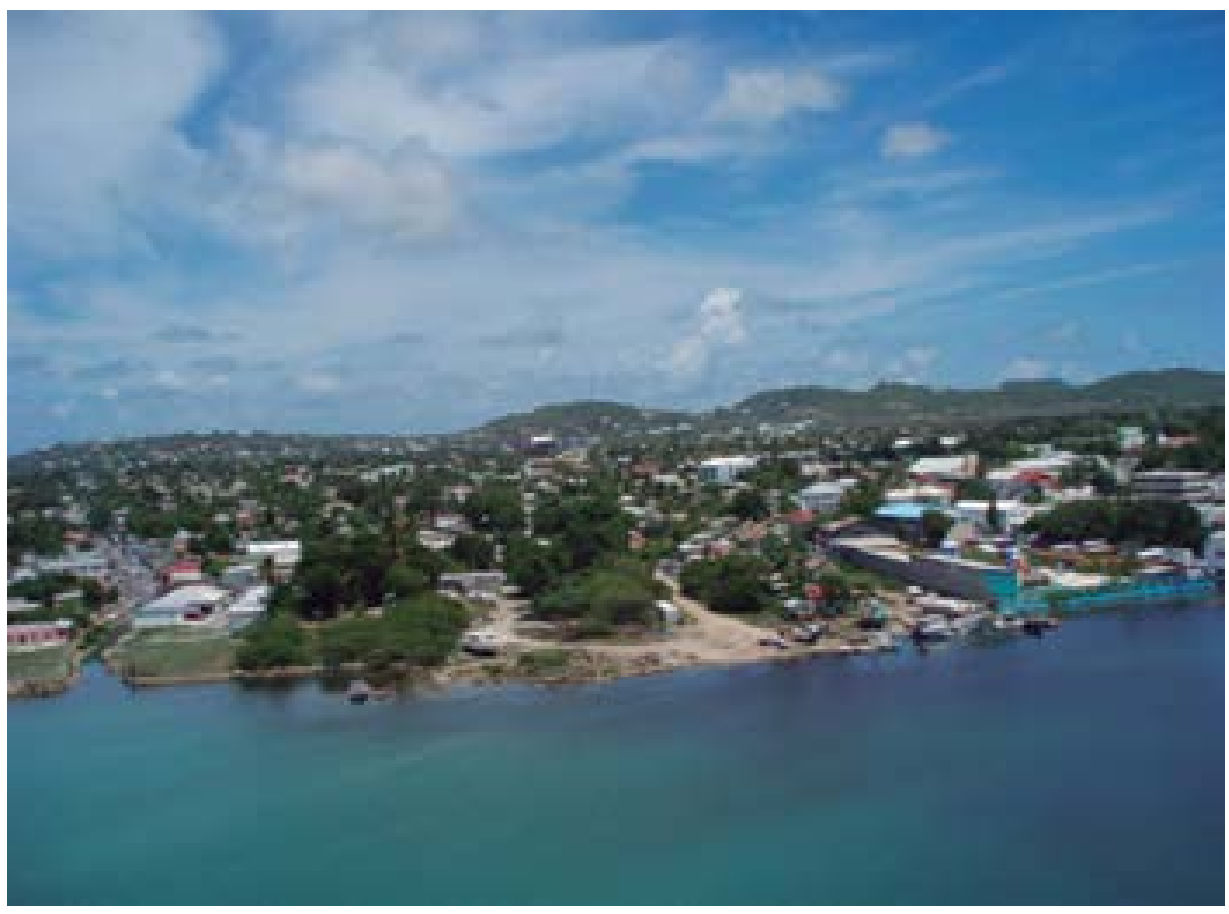

Figura 1. Vista parcial de St. John's, capital de Antigua y Barbuda. Aparte de la palpable reducida altitud de esta zona, la imagen pone de manifiesto la dificultad de establecer límites entre sectores económicos, lo urbano y lo natural, o el litoral y el interior. Fuente: Fotografía tomada por el autor en septiembre de 2007.

de tamaño pequeño, los diferentes ámbitos de actuación y sujetos del ordenamiento territorial se encuentran demasiado ligados entre sí y en muchos casos no conviene segmentar políticas de un modo rígido. Por este motivo, en el ámbito que nos ocupa es importante enfatizar en que los planes sectoriales descoordinados desde niveles administrativos inferiores no son la mejor manera de abordar los problemas. En efecto, los estudios previos deberán establecer las prioridades en las líneas de 
actuación, atendiendo al conjunto insular y no a puntos concretos del territorio, pues en ocasiones, en virtud del tamaño, resulta irrelevante acotar áreas bajo criterios de separación de ecosistemas en los que no quedan claros sus propios límites (figura 1).

Asimismo existen numerosas y diferentes formas de intervenir de manera ordenada sobre el espacio y los recursos, y por tanto no es de extrañar que el número de instrumentos sea también elevado. Es necesario, por tanto dotar de eficacia y coordinación a estos instrumentos para que realmente resulten efectivas sus acciones. La utilización de un tipo u otro suele estar relacionada con el objetivo (planificación o gestión) y la escala de trabajo, y a su vez, se pueden subdividir en dos grandes apartados según sean preceptivos o voluntarios. De esta manera nos encontramos con un amplio abanico de posibilidades en cuanto a las dimensiones del problema y los modos de enfrentarlo. Sin embargo, la implementación de este sistema integral requiere de un andamiaje legal previo que otorgue cobertura a la planificación y la gestión, además de una dotación económica realista para el correcto desempeño de las administraciones públicas.

Por tanto, se hace necesaria, la aprobación de una legislación básica a escala Estatal o insular para la gestión y planificación territorial que fomente la coordinación y la cooperación en diferentes niveles administrativos. Este planeamiento, que podríamos calificar de "cascada" o "desde arriba" (pues la jerarquía normativa emana de niveles geográficos y administrativos superiores hacia los inferiores y/o locales), debe ser la norma si se quieren evitar duplicidades en las competencias y dificultades en la implementación de las políticas públicas, pues si estas son únicamente locales, existen enormes posibilidades de que estén desacompasadas con respecto al entorno geográfico más inmediato y al conjunto insular. Igualmente es importante en este punto la cuestión de la distribución de los fondos económicos e indispensable la búsqueda de la cooperación y la coordinación internacional, por ejemplo mediante estructuras como la Alianza de Islas Pequeñas Estados (AOSIS, por sus siglas en inglés) (Nicholls y Mimura, 1998, p. 15) u otras similares.

En consecuencia, en la escala insular, se hace necesario que el ejercicio armonizador comience por implicar a todos los ministerios y órganos de gobierno con un proyecto duradero en el tiempo, pues es uno de los principales factores que incidirán en la eficacia de los resultados que 
se pretenden obtener. Por lo tanto hay que conceder atención especial al espacio y a los recursos de la isla, definiendo un proyecto político específico a todas las escalas administrativas involucradas e idealmente atendiendo también al ámbito internacional. Aquí las islas pequeñas cuentan con una gran oportunidad, pues el reducido tamaño de las administraciones públicas permite una mayor coordinación y flexibilidad que podría llegar a mitigar en parte la habitual falta de recursos económicos que padecen estos espacios.

\section{Planificación y gestión desde el punto de vista del litoral como entorno urbano}

Los criterios de delimitación de los espacios que se deben gestionar y planificar pueden ser muy variados (históricos, físico-ambientales, socioeconómicos), pero siempre suele tenerse en cuenta el funcionamiento real del territorio (Precedo, 1990), es decir las relaciones de movilidad residencia-trabajo y residencia-prestación de servicios y satisfacción de demandas sociales. La ordenación del área urbana-litoral debe tomar en consideración cuatro grandes temas:

(1) La ordenación de los asentamientos urbanos: el cual pretende evitar una excesiva densificación demográfica y constructiva. Entre las posibilidades de intervención están potenciar nuevas centralidades (es decir, dispersar actividades y equipamientos hacia las ciudades medias del área) y mantener las zonas dedicadas a usos agrarios o recreativos.

(2) La ordenación de las actividades económicas: en donde se propone su diversificación y modernización. Los objetivos serían: evitar o retrasar la desaparición de las actividades primarias; afrontar el problema de la desindustrialización, instalando nuevas actividades en áreas de vaciado de la ciudad central (industrias avanzadas y servicios muy exigentes en centralidad), en coronas metropolitanas (por ejemplo parques empresariales) y en la franja periurbana; y desconcentrar el terciario avanzado, localizado en la ciudad central, hacia los núcleos urbanos de segundo orden, e instalar actividades terciarias fuera de los núcleos urbanos (cerca del aeropuerto o de las vías de transporte).

(3) La intervención de la red de transportes: la cual debe garantizar la fluidez en las interrelaciones entre todos los núcleos del área y las conexiones con el sistema de ciudades nacional, continental, mundial. 
(4) La ordenación del espacio no urbanizado: este sufre la creciente expansión urbana, se orienta a proteger las actividades agrarias y a conservar los espacios verdes para el ocio.

Entre los problemas principales de carácter general a los que se debe atender en la planificación y gestión de este espacio se encuentran sobre todo: los relacionados con la vivienda ante un suelo escaso; los de abastecimiento y equipamiento, sobre todo de agua y energía; los problemas de tráfico que exigen de un correcto estudio destinado a la optimización de viales y el transporte público; la delincuencia y marginación social; y, por supuesto, el medio ambiente.

\section{Planificación y gestión desde el punto de vista del litoral como medio natural}

La planificación territorial debe igualmente preocuparse por la continuidad y protección de los ecosistemas. Es una labor muy complicada (ver fotografía 1), pues la vertebración del territorio mediante los transportes (e infraestructuras con impacto en el medio) es un elemento vital para la economía. El paisaje natural debe ser la clave de las políticas de ordenación, puesto que es también el elemento más frágil. Es necesario ser consciente de que la protección es importante, pero no puede quedar marginada y confinada: es necesario integrar e incrementar los ecosistemas protegidos entre sí para que existan mayores garantías para la biodiversidad. En el caso de las islas de tamaño pequeño y en términos generales, los ecosistemas naturales litorales deben ser protagonistas, sobre todo cuando existan arrecifes de coral, ya que las funcionalidades que se obtienen de ellos son irreemplazables, ya que aportan arena a las playas, funcionan como barrera protectiva natural ante dinámicas marinas violentas y además tienen valor económico específico por medio del turismo (Nurse et al, 1997, sección 9.3.3.1).

De igual modo resulta indispensable prestar atención a la singularidad de estos espacios litorales en general, pues además albergan medios de distinta naturaleza (litosfera, atmósfera, e hidrosfera salada y continental); registran un funcionamiento complejo (interacciones y cambios biológicos, geomorfológicos y químicos en períodos extremadamente breves de tiempo); contienen ecosistemas con las mayores tasas de productividad y diversidad biológica del planeta (estuarios, marismas, praderas de fanerógamas marinas); son frágiles y poseen algunas cadenas tróficas 
muy simples; son básicos como zona de cría de ciertas especies de valor ecológico y comercial; y existen unidades ambientales que cumplen una función defensiva de enorme trascendencia ante amenazas naturales como tormentas o inundaciones.

Entre los problemas principales de carácter general a los que se debe atender en la planificación y gestión de este espacio se encuentran sobre todo la degradación de los recursos naturales, la pérdida de la biodiversidad (en especial la endémica o sensible, como en el caso de los corales), el daño al patrimonio histórico-cultural costero, la disminución o dificultad de acceso al disfrute de los bienes de uso público (como las playas y la ribera del mar) y el aumento de la erosión costera.

Del mismo modo, se hace necesario reconocer algunas de las causas de los problemas mencionados o cualquier otro que se identifique. Uno de los principales causantes de problemas que afectan a los recursos es la excesiva urbanización del espacio litoral, ya sea con fines de expansión de las ciudades o para el desarrollo de actividades ligadas al turismo o al ocio. Otro es la sobreexplotación de los mismos, ya sea por técnicas inadecuadas o por no ser discriminatorias (pesqueros, forestal, acuíferos, etc), o la persistencia de ciertas actividades incompatibles con la conservación del medio y sus recursos.

También se debe destacar la creciente artificialización del borde costero con ciertas obras de ingeniería, o la construcción de infraestructuras tan importantes y exigentes en terreno como los embalses, con todos los impactos que estos traen consigo. Igualmente es importante destacar el daño que produce el uso de productos nocivos para la vida, y los vertidos de residuos. Por último, es indispensable evitar un diseño de obras o emplazamiento de construcciones inmobiliarias inadecuado, accesos deficitarios, privatizaciones y apropiaciones indebidas de patrimonio público y cultural, etcétera.

\section{Conclusiones}

Las islas pequeñas, "independientemente de la metodología empleada, son constantemente identificadas en los estudios como una de las áreas de mayor riesgo [...] y por tanto, la identificación e implementación de medidas efectivas de adaptación son sumamente importantes" (Nurse y Sem, 2001, p. 846). Los retos a los que se enfrentan estos espacios son demasiado difíciles de abarcar para muchos de ellos, pues su 
vulnerabilidad los sitúa en una posición de indefensión manifiesta frente a contingencias que otros territorios pueden afrontar sin mayores problemas. Aunque la lista de adversidades que producen grandes problemas a las islas de tamaño pequeño es larga, algunas relacionadas con el llamado cambio climático ponen en peligro de manera muy directa el futuro de muchas personas en un plazo y unos términos demasiado imprecisos como para que se puedan sacar conclusiones científicamente seguras acerca de cómo actuar en cada caso.

En este sentido, dada la variabilidad de amenazas a las que se deben enfrentar, pero sobre todo debido a las características de fragilidad de los espacios insulares de tamaño pequeño, se hace necesario que estas medidas de adaptación se lleven a cabo por medio de un plan integral que estructure desde arriba a todas las administraciones en sus políticas sectoriales que se ocupen de regir sobre los aspectos que se estimen como indispensables transversalmente. Esta manera de gestión y planificación territorial requiere un importante esfuerzo económico y organizativo inicial, pero es importante para estos espacios comprender que la solución real requiere de una aproximación integral global e incluso una mayor implicación a nivel internacional. Indudablemente, esta es la vía más adecuada de actuación en unos territorios en los que la falta de recursos de todo tipo convierte en indispensable la armonización organizativa. Además, la visión global a escala insular es la única que puede coordinar eficazmente la gestión y ordenación del espacio en torno a unos parámetros priorizados por medio de una dotación financiera realista y una ley adecuada.

\section{Referencias}

Barragán-Muñoz, J.M. (2004). Las áreas litorales de España. Del análisis geográfico a la gestión integrada. Barcelona: Ariel. Ir a libro: http://hum117.uca.es/grupogial paginaspublicaciones/ areaslitoralesdeespana

Creel, L. (2003). Ripple Effects: Population and Coastal Regions. Population Reference Bureau. Ir a libro: http://www.prb.org/pdf/ RippleEffects_Eng.pdf 
Doménech, J.L. y Sanz, F.J. (2010). Guía para la implementación de un sistema de gestión integrada de zonas costeras. La Coruña, España: Netbiblo. Ir a libro: http://www.jdomenech.com/articulos/ libro-2-GIZC.pdf

Fernández, R. (2011). La dimensión económica del desarrollo sostenible. Alicante, España: Editorial Club Universitario. Ir a libro: http://www. editorial-club-universitario.es/libro.asp?ref=4494

Gómez, D. (2001). Ordenación Territorial. Madrid: Editorial Agrícola Española. Ir a libro: http://www.editorialagricola.com/v_portal/ productos $/$ productoverasp $? \operatorname{cod}=33 \&$ te $=38 \&$ idage $=81 \&$ vap $=0$

Hay, J. (2000). Climate Change in the Pacific: Science-Based Information and Understanding", en Guillespie, A., and Burns, W. (Eds.) Climate Change in the South Pacific: Impacts and responses in Australia, New Zealand and small island States (p. 285). Dordrecht: Kluwer Academic Publishers.

Hinrichsen, D., (1998). The coastal population explosion. The next 25 years: Global issues. National Oceanic and Atmospheric Administration. Recuperado de: http://oceanservice.noaa.gov/ websites/retiredsites/natdia_pdf/3hinrichsen.pdf

Leatherman, S. P. (1997). Beach ratings: a methodological approach. Journal of Coastal Research, 13(1), 253-258. Ir a artículo: http:// journals.fcla.edu/jcr/article/view/80128/77387

Mimura, N. y Nunn, P. D. (1998). Trends of Beach Erosion and Shoreline Protection in Rural. Fiji. Journal of Coastal Research, 14(1), 37-46. Ir a artículo: http://journals.fcla.edu/jcr/article/view/80545/77768

Mimura, N., y Nurse, L. A. (2007). Small Islands. En Parri, M. L., Canziani, O. J., Palutikof, J. P., van der Linden, P. J., \& Hanson, C. E. (Eds), Climate Change 2007: Impacts, Adaptation and Vulnerability (687-716). England: Cambridge University Press. Recuperado de: http://www.ipcc.ch/publications_and_data/ar4/ wg2/en/contents.html 
Nicholls, R.J. y Mimura, N. (December, 1998). Regional issues raised by sea-level rise and their policy implications. Climate Research, 11, 5-18. doi: http://dx.doi.org/10.3354/cr011005

Nurse, L. A., y Sem, G. (2001). Small Island States. En McCarthy, J. J., Canziani, O. F., Leary, N. A., Dokken, D. J., White, K.S. (Eds). Climate Change 2001: Impacts, Adaptation and Vulnerability. Contribution of Working Group II to the Third Assessment Report of the Intergovernmental Pannel on Climate Change (843-876). England: Cambridge University Press. Ir a libro http://www.ipcc. ch/ipccreports/sres/regional/index.php?idp $=0$

Nurse, L. A., McLean, R. F., \& Suarez, A. G. (1997). Small Island States. En Watson, R. T., Zinyowera, M. C., Moss, R. H. (Eds). The Regional Impacts of Climate Change: An Assessment of vulnerability (331-354). England: Cambridge University Press. Ir a libro: http://www.cambridge.org/us/

Precedo, A. (1990). La red urbana. Madrid: Síntesis.

UN-OHRLLS (2013). UN Office of the High Representative for the Least Developed Countries, Landlocked developing countries and Small Islands Developing States. Recuperado de: http://www. unohrlls.org/ 